\title{
Manteniendo lazos vía Web: El Caso de las Comunidades Mexicanas de Emigrantes en los EUA
}

\author{
Víctor M González \\ Manchester Business School, University of Manchester, United Kingdom \\ $<$ vmgonz@manchester.ac.uk > \\ Luis A. Castro \\ Manchester Business School, University of Manchester, United Kingdom \\ $<$ Luis.Castro@postgrad.manchester.ac.uk >
}

\section{Introducción}

La migración es un fenómeno social que manifiesta sus efectos en las estructuras económicas, marcos jurídicos, esquemas sociales y aun los aspectos mas cotidianos de las comunidades involucradas. Países como Estados Unidos de América (EUA), Canadá, Reino Unido y Australia son áreas típicas de inmigración y los flujos migratorios a estos destinos se han ido incrementando continuamente desde mediados del siglo XX (Castles and Miller, 2003). Las razones para migrar son numerosas desde la búsqueda de trabajo o asilo político hasta el emprender oportunidades comerciales o adquirir educación y conocimientos especializados.

Este trabajo se concentra en particular en el contexto migratorio de México a EUA, el cual ha sido considerado uno de los flujos migratorios más importantes a nivel mundial en cuanto incentivado por la búsqueda de oportunidades laborales. La inmigración mexicana en los EUA se ha estado incrementando en los últimos años, y ha pasado de concentrarse en algunas areas como los estados de California o Texas, ha cubrir prácticamente el pais por completo (Zúñiga and Hernández-León, 2005).

Como resultado de la distancia que los separa, los emigrantes se comunican de manera habitual como una manera de mantener la cercanía con su familia y comunidades de origen. Por muchos años, la principal forma de contacto fueron el correo convencional y las llamadas por teléfono. Sin embargo, la disponibilidad de Tecnologías de Información y Comunicación (TIC), en particular Internet, ha facilitado la creación de sitios Web comunitarios que ofrecen a los emigrantes información así como oportunidades de estar en contacto con la gente de sus comunidades de origen.

Este artículo ofrece un análisis de los sitios Web de comunidades mexicanas diseñados específicamente con el objetivo de proporcionar un medio de comunicación a aquellos miembros de la comunidad viviendo lejos de ella, principalmente en los EUA. Nuestra investigación busca identificar la presencia en Internet de esta clase de sitios en Internet así como también proveer una caracterización inicial con respecto al tipo de apoyo que brindan a los miembros de esas comunidades viviendo en el extranjero. Nuestras conclusiones buscan ser un punto de partida para hablar de cómo se puede incrementar el sentido de 'conexión' que los emigrantes tienen hacia sus comunidades a través de nuevas formas de interacción usando diferentes tecnologías y, de igual forma, facilitar su entera participación en la vida de sus comunidades de origen.

De manera inicial, y como un ejercicio de reflexion que motivó este trabajo, nuestra propia experiencia como emigrantes de comunidades relativamente pequeñas nos sugirió que nuestro primer contacto con México, ciertamente es a través de nuestras familias, pero fue a través de estos sitios Web que pudimos conectarnos con la vida cotidiana de nuestras comunidades de origen. Previo a nuestro esfuerzo, se sabía de un número indefinido de sitios Web comunitarios usados para comunicar a los emigrantes mexicanos en EUA con sus familias y comunidades de origen. En el pasado, la prensa ha reportado evidencia anecdótica acerca de los servicios provistos por esos sitios, los cuales actúan como puentes para aquellos que viven lejos. Nuestra motivación surge entonces de entender que el tipo de sitio web que deseabamos estudiar no cubre una dimension nacional que puede ser importante para el migrante, pero provee otra clase de acercamiento a aquellas redes sociales que por la naturaleza misma de las comunidades parten de un universo mas pequeño y en las que el impacto que el Internet así como las implicaciones de estar comunicados pueden tener alacanzar otros objetivos.

El articulo está organizado de la siguiente manera: en la siguiente sección se presenta una discusión sobre la 
naturaleza de las comunidades dispersas ${ }^{1}$ dentro del contexto migratorio México-USA. Esta sección provee una imagen acerca de la magnitud del fenómeno en este contexto en particular. En la siguiente sección, se habla acerca de la importancia del rol de las Tecnologías de Información y Comunicación (TIC) como puente para conectar a los emigrantes y sus comunidades. Después de esta sección se presenta una sección que discute y analiza los resultados de otros estudios que se han llevado a cabo sobre comunidades dispersas señalando la relevancia de las TIC y la forma en que la gente las ha adoptado como una forma de acercarse a sus familiares, comunidades y su propia identidad. Enseguida se presenta el análisis de los sitios Web de comunidades en México y se presentan, en particular, cuatro ejemplos de estos sitios Web. Finalmente, se presentan la discusión y conclusiones del estudio.

\section{La Naturaleza de las comunidades dispersas en México}

La migración es un fenómeno complejo que está alterando tanto a la sociedad como a los gobiernos alrededor del mundo. Durante el siglo pasado, la migración, en su mayoría, de la clase trabajadora mexicana hacia los EUA ha sido considerable. Casi siempre, las regiones con menos oportunidades, que carecen de trabajos bien remunerados $\mathrm{u}$ oportundiasdes para obtener educación de calidad, son las que enfrentan este tipo de movimientos. De acuerdo al Consejo Nacional de Población en México (CONAPO), hay más de 10 millones de personas nacidas en México, viviendo en los EUA (CONAPO, 2005). Estos movimientos masivos de gente son conocidos en la literatura como comunidades en Diaspora, lo cual se refiere a personas que viven alejadas de sus lugares de origen. En el caso particular de los mexicanos viviendo en el extranjero, en EUA en particular, Rinderle (2005) concluye en su trabajo que son una comunidad en Diaspora ya que han experimentado: 1) un desplazamiento físico histórico, 2) desarticulación e hibridez cultural, 3) añoranza por la patria, 4) desplazamiento estructural y una relación compleja entre el estado-nación y la Diaspora, 5) alienación de su 'nueva patria', y 6) una identidad colectiva definida por la relación entre su patria y su nueva patria.

Dejando de lado el debate acerca de las condiciones que crean este fenómeno social, es un hecho que estos flujos migratorios han contribuido a la revitalización de la fuerza de trabajo en EUA (Zúñiga and Hernández-León, 2005). De igual forma, las comunidades de origen de los emigrantes han estado experimentando un cambio profundo en lo social y lo económico. Es inegable, que muchas de esas economías así como proyectos comunitarios dependen altamente de las remesas enviadas por la gente que vive en el extranjero. De acuerdo al Bando de México, el banco central, en el 2006m los mexicanos viviendo en el extranjero enviaron mas de \$23,000 millones de dólares en remesas (BANXICO, 2006), las cuales se usan principalmente para consumo familiar y son, en la práctica, una de las estrategias de desarrollo más efectivas ya que el dinero llega a donde existe mayor necesidad (Merz, 2005). De igual forma, las asociaciones de inmigrantes mexicanos en EUA, formadas por personas de la misma comunidad de origen, se están convirtiendo en entidades cada día más organizadas con una influencia política y económica considerable en sus comunidades. Sus actividades se dirigen principalmente a proyectos comunitarios tales como acciones altruistas y embellecimiento de su comunidad (Merz, 2005).Dichos proyectos incluyen la restauración de iglesias o pavimentación, los cuales son patrocinados en parte por las asociaciones de emigrantes formadas en el extranjero y en parte por los gobiernos locales (a nivel municipal o estatal)..

Sin embargo, mas allá de los beneficios económicos, la migración tiene consecuencias no siempre positivas para los emigrantes, sus familias así como sus comunidades. Por ejemplo, los periodos tan extensos de ausencia puede hacer propensos a los individuos a experimentar sentimientos de nostalgia, los cuales pueden obstruir su adaptación a su nuevo hogar (Fisher, 1989; van Tilburg and Vingerhoets, 1997). De igual forma, las familias mexicanas, tradicionalmente muy apegadas, se ven afectadas por largos periodos de separación que, en algunos casos, puede resultar en niños frágiles emocionalmente o, algunas veces, puede llevar a una separación permanente. Además, las comunidades experimentan algunos retos como lo es la disminución de la población y la falta de mano de obra lo que crea círculos negativos, en los que la comunidad llega a una posición en la cual no puede retener a sus miembros porque no tiene oportunidades, pero no puede crear oportunidades porque carece de suficiente miembros. Este ultimo problema es más notable en comunidades rurales donde la población crece a un ritmo lento o, algunas veces, hasta decrece debido a la migración de familias enteras (CONAPO, 2006b).

Las comunidades de inmigrantes mexicanos en EUA pueden también ser descritas como comunidades trasnacionales, las cuales son "caracterizadas por la incorporación de la cultura de migración (y remesas) en el tejido del sistema social, tanto que la gente vive entre dos mundos: Norteamérica y sus comunidades de origen en México" (Conway and Cohen, 1998). El principal reto que enfrentan estas comunidades es el que viven dos realidades: por un lado lidiando con las preocupaciones diarias en sus nuevos hogares tal como problemas relacionados al trabajo, salud, licencias de manejo, la escuela de sus hijos y hasta problemas de alienación o discriminación y, por otro lado, la angustia por problemas regionales o nacionales en México que pueden afectar potencialmente a su familia así como a su comunidad.

1 Comunidad diaspórica se refiere a la gente que vive en el extranjero y que son de la misma comunidad de origen; por comunidad dispersa se refiere a la gente de una misma comunidad de origen dispersa en todo México y en el extranjero. 


\section{Manteniendo lazos con la patria: el rol de las TICs}

Como resultado de la distancia física que los separa, las familias se mantienen en contacto regularmente para buscas mantener la cercanía. Por muchos años, la principal forma de contacto de los inmigrantes era el correo convencional así como las llamadas telefónicas. Sin embargo, este comportamiento ha ido cambiando gradualmente debido a la cada ves más común disponibilidad de computadoras con acceso a Internet aun en lugares remotos. De acuerdo a la Asociación Mexicana de Internet, en abril de 2007, 22.7 millones de personas tenían acceso a Internet, de los cuales el 55\% lo hacian desde su hogar (AMIPCI, 2007). Esta penetración se ha incrementado en parte debido a que los equipos computacionales se han vuelto más accesibles a través de planes crediticios e iniciativas de gobierno para promover el acceso a Internet desde centros comunitarios (Salazar, 2004). Por otra parte, aunque no se han realizado estudios específicos, un reporte publicado en el año 2000 indica que, después de los asiáticos, los latinos ocupan un segundo lugar en la adopción de Internet en el hogar (Walsh, 2000), no obstante, estos números son todavía reducidos en comparación con otros subgrupos en los EUA como lo son los blancos y afro-americanos (Fox and Livingston, 2007). Tomando en cuenta eso, y el hecho de que el equipo computacional y las tarifas de acceso a Internet son mas baratos en EUA, se puede decir que se esta haciendo mas factible para cualquier inmigrante mantenerse en contacto con sus familias en México ya sea a través de el envio de correos electrónicos o platicar con ellos en línea vía mensajería instantánea o servicios de voz sobre IP.

La creciente disponibilidad de TICs ha facilitado también la creación de sitios Web comunitarios que proveen información local así como oportunidades de comunicación para aquellos que viven lejos de sus hogares. Durante algunos años, la prensa y otros medios de comunicación han estado reportando acerca del fenómeno, destacando el rol que estos sitios Web están jugando en mantener a las comunidades y sus familias unidas (Gonzalez-Mendez, 2002). Se puede argumentat que la presencia de estos sitios Web tiene el potencial de incrementar el sentido de conexión de los migrantes en el extranjero hacia sus comunidades asi como de reforzar su apegamiento a ellas, haciendo posible el involucrarse en asuntos sociales, políticos y económicos. Nuestro esfuerzo esta enfocado a ir más allá de la evidencia anecdótica, y llevar a cabo un estidio que analice esta clase de sitios Web comunitarios y el impacto que los servicios que proveen pueden tener para aquellos que viven en el extranjero.

\section{Otras comunidades dispersas usando TIC}

A medida que la gente migra, se incrementa la necesidad de comunicarse y mantenerse al tanto de las cosas que pasan en sus lugares de origen. Esta sección ofrece una reseña de investigación llevada a cabo con el objeto de estudiar el rol que juegan las TICs en las vidas de las comunidades dispersas.

Uno de esos casos en los cuales el Internet ha servido para conectar a comunidades dispersas se reporta en un estudio etnográfico llevado a cabo por Bernal acerca del uso de Dehai.org, un ciberespacio público, por eritreos en Diaspora (Bernal, 2005). Dehai fue iniciado por la Diaspora eritrea que vive en EUA con el objetivo de crear un espacio público para el debate y la expresión de ideas acerca del conflicto entre Eritrea y Etiopía (1998-2000). Dehai.org está compuesto principalmente por foros y boletines informativos, lo cual hizo posible la inclusión de eritreos ordinarios en discusiones de asuntos de interés nacional. Bernal descubrió que eritreos en diáspora usaron las discusiones en línea para ponerse en contacto con otros eritreos en su misma condición y mantenerse al tanto de lo que pasaba en Eritrea y, finalmente, crear un espacio público transnacional eritreo que se extiende más allá del ciberespacio. Bernal concluye que los eritreos fueron capaces de crear una comunidad con un sentido de pertenencia y de identidad difícilmente alcanzado por otro espacio público en Eritrea (Bernal, 2005).

Otro de estos casos de Diásporas que usan TICs para mantener el sentido de conexión es el caso de la diáspora de Trinidad y Tobago. Millar y Slater llevaron a cabo un estudio etnográfico acerca del uso de Internet por parte de los trinitarios. El objetivo del estudio era el de averiguar cómo es que ellos adaptaban los medios de comunicación para hacerse la idea de que estaban encasa (Miller and Slater, 2000). La investigación consistió en un estudio in-situ en Trinidad y Tobago así como también entrevistas con trinitarios viviendo fuera de su país con el objetivo de entender que significa ser un trinitario. También se llevaron a cabo entrevistas y cuestionarios para caracterizar el uso y penetración del Internet en Trinidad y Tobago. El estudio se centró principalmente en cuatro asuntos: negocios, afinidad, consumo e identidad. Sus resultados indican que el Internet puede ayudar a reforzar el sentido de identidad y singularidad de una cultura en particular. Miller y Slater concluyen que en el caso de los trinitarios que viven en diáspora, el Internet ha ayudado a reforzar considerablemente los lazos familiares, ayudando a mantener relaciones más cercanas entre padres e hijos así como entre hermanos.

Un tercer caso relacionado es el de los emigrantes canadienses originarios de Newfoundland quienes han estado manteniendo lazos con su hogar y con otros que viven en diáspora a través de TIC (Hiller and Franz, 2004). Aun cuando el trabajo de Hiller y Franz presentan el caso de migración interna en Canadá, sus conclusiones pueden ser usadas para resaltar algunos de los aspectos relevantes para casos de migración internacional. En particular, ellos contrastan el uso y el rol de las TIC en diferentes etapas de la migración: pre-emigrante, post-emigrante y emigrante establecido. Durante la etapa pre-emigrante, debido a que el emigrante aun no cambia de residencia, las TIC juegan un rol instrumental en el que su propósito es informar acerca del lugar al que se quieren trasladar. La etapa post-emigrante es cuando el emigrante ha estado fuera de su lugar de origen por un lapso menor a 5 años. Durante esta fase, las TIC ayudan a 
facilitar el contacto a casa así como establecer contacto con otros emigrantes. Finalmente, durante la etapa donde se encuentran establecidos, los que tienen 5 años o mas, ellos encontraron que las TIC ayudan a conservas la identidad así como a revivir los aspectos culturales de su comunidad. En esta etapa, los emigrantes están tratando de redescubrir su conexión con sus comunidades así como sus raíces.

Estos tres casos son ejemplos de cómo la tecnología ha sido usada para facilitar una mejor identificación y apego a sus comunidades así como el mantenimiento de las relaciones familiares para aquellos que viven más allá de las fronteras. Sin embargo, si se compara con el caso de los trabajadores emigrantes mexicanos, estas diásporas difieren claramente en algunas características fundamentales. Particularmente, en contraste con el contexto mexicano, Eritrea era una nación en conflicto, lo que pudo influenciar seriamente la consolidación de Dehai.org como espacio público para debatir y ponerse al tanto de lo que pasaba. Además, los estudios sobre eritreos y trinitarios ilustran casos que se centran en naciones enteras en vez de comunidades pequeñas. Sin duda, los inmigrantes mexicanos en los EUA tienen un interés particular en su país, pero creemos que esos sentimientos pueden ser aun más fuertes hacia su comunidad de origen donde residen su familia, amigos y conocidos. En particular, el estudio de la comunidad de Newfoundland en Canadá es quizás el que refleja más la necesidad de reconectarse con el lugar de origen a nivel comunitario. Nosotros argumentamos que de hecho, es a este nivel, en donde las persona tiene más posibilidades de volverse activo política, social y económicamente.

\section{Estudio de sitios Web comunitarios}

Esta sección presenta los métodos usados en la obtención de datos, así como las técnicas para el análisis y las conclusiones del estudio exploratorio de sitios Web hechos con el objetivo de sustentar y conectar emigrantes originarios de municipios en México con altos niveles de migración hacia los EUA.

\section{Metodología de investigación}

Las siguientes preguntas de investigación motivaron nuestro estudio.

1. ¿Cuál es la presencia real en Internet de las comunidades en México con (muy) altos niveles de migración?

2. ¿Qué clase de funcionalidad y/o servicios de información caracterizan a los sitios Web de comunidades dispersas mexicanas?

3. ¿Cuáles son los principales servicios que proveen y que necesidades satisfacen estos sitios?

Para resolver estas preguntas, se inició con la identificación de las comunidades en México con alto o muy alto nivel de migración. Basamos esta identificación en los datos proporcionados por CONAPO, quien creó un índice con el objeto de clasificar los estados y municipios en cinco clases -muy alto, alto, medio, bajo, muy bajo- que indican el grado de migración basado en cuatro indicadores: hogares que reciben remesas, hogares con emigrantes que dejaron el país en los últimos 5 años, hogares con emigrantes que dejaron y regresaron al país en los últimos 5 años y, finalmente, hogares con miembros de la familia que regresaron y que habían migrado antes de 1995. Tomando en cuenta este índice, fue posible identificar 472 municipios de 2,433 que tiene México tienen alto o muy alto nivel de migración (CONAPO, 2006a).

Los municipios que fueron identificados después de esta selección comparten ciertas características. Como era de esperarse, estos municipios pertenecen a estados con niveles más altos de migración, los cuales tienden a ser estados del sur de México como lo son Guerrero y Michoacán o los estados del bajío mexicano como lo son Jalisco, Guanajuato y Zacatecas. De la misma forma, tienden a ser comunidades pequeñas con un promedio de 3,838 hogares (d.e. 4,354).

Partiendo de un universo inicial de 472 municipios, realizamos una búsqueda en Internet usando Google como máquina de búsqueda. Usamos el nombre del municipio (por ejemplo, "Atotonilco", "San Juan de los Lagos", "Tequesquiapan") como palabra clave en la búsqueda. El propósito era identificar si estos municipios contaban con un sitio Web en línea. Enseguida visitamos cada página que parecía indicar que era de un sitio Web comunitario. Nos centramos en los primeros 30 resultados de la búsqueda porque después de algunos intentos nos dimos cuenta que si un nombre de dominio estaba registrado o estaba hospedado en algún otro servidor como Grupo de MSN o Grupos de Yahoo casi siempre aparecía en los primeros 12 resultados. De esta manera, al analizar los primeros 30 resultados nos daba la confianza de no perder un sitio relevante. Aun cuando muchos de los resultados eran de sitios gubernamentales, presentaban información que no estaba relacionada o con el objetivo de dar sustento a las comunidades dispersas. Tal información incluía el CV del presidente municipal, programas sociales, finanzas y organigrama gubernamental. El criterio que utilizamos para decidir si era un sitio de una comunidad dispersa era que un sitio era válido si su propósito era el de conectar a aquellos que viven alejados con los que viven en la comunidad. Los principales aspectos para identificar este tipo de sitios eran la información publicada por el administrador (webmaster), el contenido o los tipos de funcionalidades ofrecidas. Los datos se recolectaron y validaron por ambos autores en el verano del 2006.

Una vez que se identificaron los sitios, se procedió a analizar cada uno de ellos para clasificar sus principales características funcionales. Se inició con algunos de los sitios, y se definieron un conjunto inicial de posibles 
funcionalidades. Este conjunto se fue redefiniendo hasta que todos los sitios Web fueron analizados y dicho conjunto contenía las características comunes de todos los sitios.

El análisis continuó usando un análisis mas detallado del contenido de una muestra de cuatro sitios. Estos sitios se seleccionaron ya que representaban buenos ejemplos de las características observadas, es decir, incluían una buena variedad de servicios además de ser muy activos. Dos de los sitios eran del estado de Zacatecas (el estado en México con el nivel de migración más alto), uno de Guerrero y uno del estado de Michoacán. Cabe señalar que los autores no son originarios de ninguno de esos estados, y por consiguiente de los municipios, a los que los sitios pertenecen. Este análisis sirvió para identificar las limitaciones de las tecnologías actuales así como detectar oportunidades para nuevos sitios comunitarios.

\section{Resultados}

Los resultados generales de nuestra búsqueda de sitios comunitarios es que cerca del 20\% (94) de los sitios con alto y muy alto índice de migración cuenta con sitios con el objetivo de conectar aquellos que viven fuera de la comunidad con los que viven en ella. Se descubrieron un total de 114 sitios ya que algunos de los municipios cuentan con más de uno. Además, la mayoría de los sitios $(71 \%)$ cuenta con su propio nombre de dominio, el cual es generalmente el nombre del municipio y están en servidores privados, pagados por los webmasters, mientras que hay otros que están en servidores 'públicos' como lo son espacios de MSN o Grupos de Yahoo. También se encontró que, de acuerdo a comentarios publicados por el webmaster (por ejemplo en la sección 'Acerca de...'), los sitios fueron iniciados por personas con conocimientos técnicos, aun cuando actualmente la mayoría de ellos se mantienen vivos por la participación de los usuarios.

\section{Funcionalidad de los sitios Web comunitarios}

Los sitios proveen una variedad de funciones que incluyen varios servicios tales como el compartir álbumes fotográficos, información general como historia de la región, video en vivo, radio y TV local, la cocina regional y hasta cambio de moneda peso/dólar. Después del análisis de los 114 sitios Web se obtuvo un conjunto de servicios que se listan en la Tabla 1.

Tabla 1. Categorías y servicios que proveen los sitios Web

\begin{tabular}{|l|l|}
\hline Servicio provisto por el sitio Web & \% de sitios que lo proveen \\
\hline Información histórica & 62 \\
\hline Galería de fotos estática (mantenida por el webmaster) & 51 \\
\hline Foros & 49 \\
\hline Libro de visitas & 44 \\
\hline Salas de Chat & 43 \\
\hline Publicidad de negocios locales & 35 \\
\hline Galería de fotos dinámica (mantenida por usuarios) & 32 \\
\hline Fotos del Municipio & 31 \\
\hline Comentarios de la Editorial & 28 \\
\hline Noticias locales & 27 \\
\hline Encuestas & 24 \\
\hline Videos del municipio & 18 \\
\hline Condiciones climatológicas del municipio & 16 \\
\hline Control de acceso con contraseña & 16 \\
\hline
\end{tabular}




\begin{tabular}{|l|l|}
\hline Estaciones de radio & 15 \\
\hline Anuncios clasificados & 14 \\
\hline E-mail comunitario & 11 \\
\hline Calendario & 10 \\
\hline Tablero de mensajes rápidos & 10 \\
\hline Cocina regional & 7 \\
\hline Juegos & 6 \\
\hline Listas de e-mail & 5 \\
\hline Anuncio de cumpleañeros & 4 \\
\hline Entrevistas a gente local & 3 \\
\hline Envío de SMS y desplegados en la pagina & 3 \\
\hline Video cameras en vivo & 2 \\
\hline Tarjetas postales & 2 \\
\hline Presentaciones acerca del municipio & 1 \\
\hline Estación de TV & 1 \\
\hline Cambio de Moneda & 1 \\
\hline Árboles genealógicos & \\
\hline
\end{tabular}

Partiendo de la idea que estos sitios tienen el fin de representar a sus comunidades en el Internet, una parte significante de ellos incluyen información histórica así como la localización geográfica. De manera similar, muchos de los sitios enfatizan la comunicación proveyendo servicios que varían desde libros de visitas, Chat en línea y tableros de mensajes. Además, espacions para compartir fotos son otro de los componentes importantes que identificamos, el cual en mucho casos se cuenta con una herramienta para publicar fotos de manera directa o bien que sean publicadas con la intervención del webmaster.

La funcionalidad provista se puede agrupar en cinco grandes grupos: (1) herramientas de comunicación, (2) exhibición de media, (3) archivo para consultar información, (4) información local actual y (5) comercio. La Tabla 2 muestra los porcentajes de sitios que ofrecen estos servicios basados en la clasificación previamente descrita. La categoría de Herramientas de Comunicación incluye los tableros de mensajes, fotos libro de visitas, salas de Chat, etc. La categoría de Exhibición de Media incluye las galerías de fotos, videos así como presentaciones. La categoría de Información Local incluye cámaras en vivo, enlaces a estaciones de radio y televisión, noticias locales y las condiciones climatológicas. Finalmente, la categoría de Comercio comprende publicidad, avisos clasificados e información del cambio de moneda.

Tabla 2. Agrupación de los servicios de los sitios Web comunitarios

\begin{tabular}{|l|l|}
\hline Funcionalidad del sitio web & \% de sitios que lo proveen \\
\hline Herramientas de comunicación & 92.5 \\
\hline Exhibición de Media & 89.4 \\
\hline Archivo de Consulta & 76.6 \\
\hline
\end{tabular}




\begin{tabular}{|l|l|} 
Información Local & 69.1 \\
\hline Comercio & 38.3 \\
\hline
\end{tabular}

Tal como lo muestra la Tabla 2, la mayoría de los sitios Web cuenta con información acerca del municipio, sus origines, historia y personas sobresalientes de la comunidad. Esto, en cierta forma, ayuda a alcanzar el objetivo de presentar su comunidad al resto del mundo. Sin embargo, más allá de este objetivo, los sitios tienen una función comunicativa. A través de los servicios que ofrecen, los sitios proveen a la gente de la comunidad un lugar de reencuentro y saber acerca del paradero de viejos amigos y familia. Muchos de los mensajes publicados en los foros y libros de visitas tenían el propósito de reestablecer los lazos sociales. Aun los libros de visitas, cuyo propósito es el de dejar notas para los administradores del sitio, eran usados para publicar mensajes que solicitaban ser contactados por personas que los conocieran, también se publican mensajes con regularidad para obtener información de alguien en especifico que no han visto en mucho tiempo.

Aunque la creación de contenido en muchos de los sitios se lleva a cabo por una sola persona (o algunos pocos), en un importante número de ellos (32\%) la creación de contenido es a través de los usuarios quienes son los que publican su propio contenido. Muchos de los sitios moderan el contenido antes de ser publicado, pero aun así, este modelo es más abierto y su adopción puede resultar en diferentes dinámicas con respecto a compartir experiencias. De esta forma, la responsabilidad de publicar contenido no recae solo en los administradores sino también en los usuarios.

Finalmente, algunos de los aspectos de la vida de estas comunidades se ven reflejados a través de algunos de estos servicios. Algunos sitios proveen videos de festividades, peregrinaciones, carnavales o desfiles. Unos pocos también dan acceso a cámaras en vivo de modo que la gente pueda sentirse aun más cerca de los eventos cotidianos al momento en que suceden. Además, la gente puede acceder a información tal como el clima local o enlaces a estaciones locales de radio y/o televisión, lo cual puede darles una cierta sensación de cercanía a ciertas situaciones.

Tabla 3. Características de los cuatro municipios

\begin{tabular}{|l|l|l|l|l|}
\hline Municipio & Tarandacuao & Arcelia & Juchipila & Moyahua \\
\hline Estado & Guanajuato & Guerrero & Zacatecas & Zacatecas \\
\hline Población & 10,252 & 31,401 & 11,603 & 4,600 \\
\hline Hogares & 2,437 & 7,056 & 3,314 & 1,293 \\
\hline Penetración de TV & $94.2 \%$ & $78.8 \%$ & $95.2 \%$ & $91.1 \%$ \\
\hline Penetración de computadoras & $7.67 \%$ & $8.16 \%$ & $16.54 \%$ & $7.35 \%$ \\
\hline Nivel educativo (años) & 6.46 & 6.60 & 6.73 & 5.61 \\
\hline Índice de migración & Alto & Alto & Muy Alto & Alto \\
\hline
\end{tabular}

\section{Cuatro instancias de los sitios Web comunitarios}

En esta sección se presentan detalles de cuatro sitios elegidos de entre los 114 sitios a manera de ilustrar como las personas usan estos sitios para mantener un cierto grado de consciencia y vinculación con los eventos que pasan en sus comunidades de origen. La tabla 3 muestra algunos datos demográficos de los municipios a los que pertenecen los sitios. La mayoría de los municipios son pequeños con un promedio de 13,600 habitantes aproximadamente. El acceso a la tecnología es bajo pero dentro de los niveles nacionales. Finalmente, todos ellos experimentan índices de migración alto o muy alto.

\section{El sitio Web de Tarandacuao (http://www.mitaranda.com)}

Tarandacuao es un municipio en el estado de Guanajuato, localizado en el centro del país, con agricultura y comercio limitados. Este municipio ha sido clasificado por CONAPO como un municipio con un alto índice de migración (CONAPO, 2006a). 


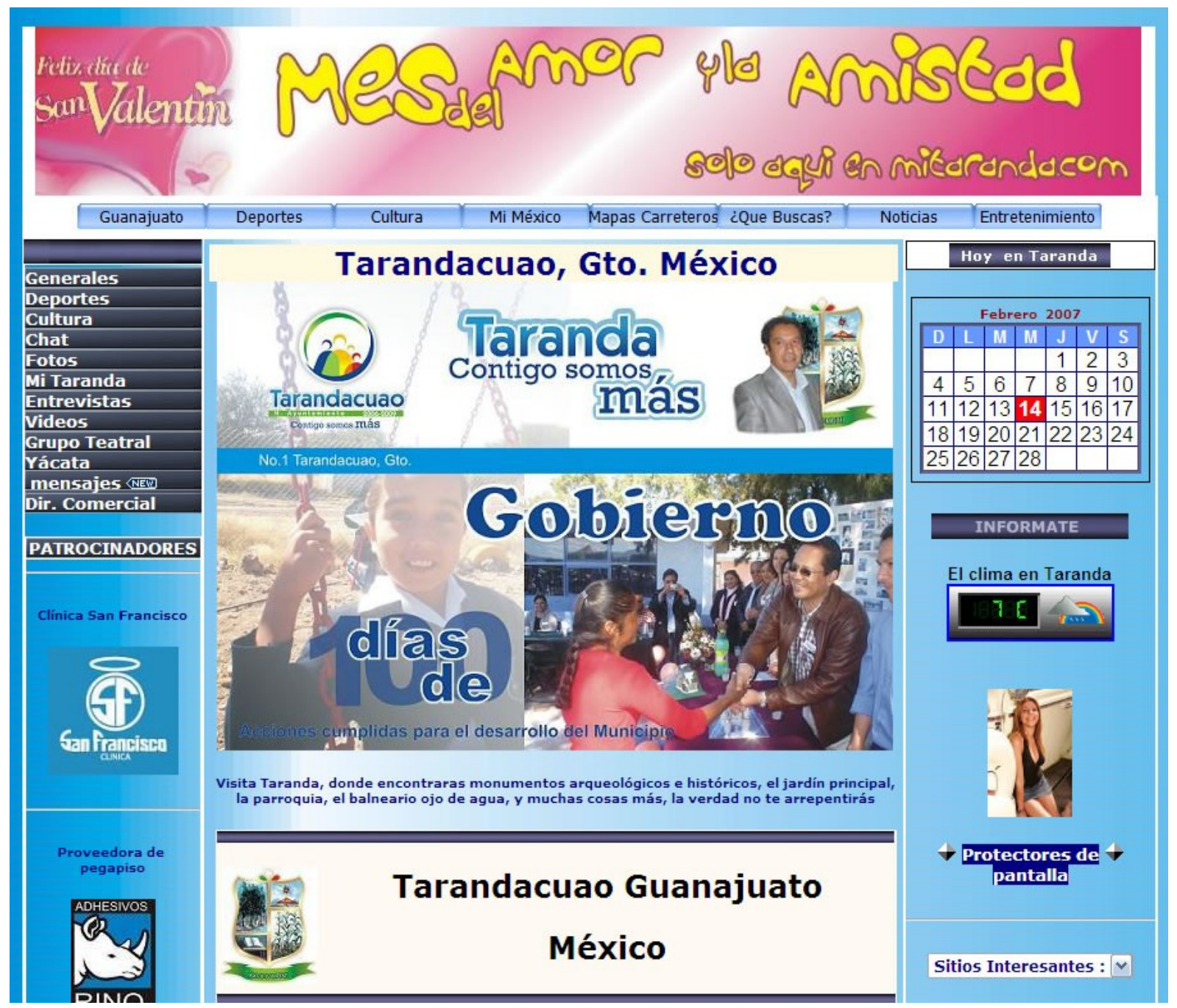

Fig. 1. Captura de pantalla del sitio Web de Tarancuao, Guanajuato

MiTaranda.com (Figura 1) es un sitio Web que pone especial énfasis en informar a los usuarios sobre cuestiones locales. Así, tanto notas informativas como nuevas fotos son publicadas de manera frecuente. El sitio también ofrece entrevistas a personajes públicos de la comunidad y ofrece, además, enlaces a servicios municipales, estatales y federales. El sitio Web no usa programación para generar páginas dinámicas e interactivas. El sitio es administrado centralmente y actualizado por el webmaster, lo cual, ciertamente, lo hace menos dinámico. Las fotos publicadas son generalmente de personas que viven fuera de la comunidad y presenta además eventos sociales tales como bodas, además de fiestas o miembros de la comunidad cuando están visitando otros lugares. El sitio cuenta además con un Chat, el cual está hospedado en un servidor externo. Finalmente, también se publica información acerca de servicios en la comunidad (e.g. personas que pinten casas o con otros oficios).

\section{El sitio Web de Arcelia (http://www.arcelia.net)}

Arcelia es un municipio en el estado de Guerrero en el sureste mexicano. Arcelia ha sido clasificado por CONAPO como un municipio con un índice alto de migración (CONAPO, 2006a).

El sitio de Arcelia provee varios servicios para los visitantes como lo es información general acerca del municipio. Este servicio clasifica la información en cuatro categorías: información general, producción económica, cultura y una sección de varios. La sección General incluye información acerca de la historia y localización del municipio dentro del contexto nacional. De manera similar, se muestran las principales carreteras por las cuales se puede llegar al municipio. Finalmente, se presenta también una breve reseña del municipio y una descripción del gobierno municipal. La sección llamada Producción Económica se refiere a las principales actividades económicas y comerciales en la región como lo son agricultura, ganadería, minería, comercio y la industria del huarache (sandalias de cuero). La tercera sección llamada Cultura es acerca de lugares para viajar dentro del municipio, gastronomía local y artesanías, danzas regionales, festividades locales y finalmente la cartelera del teatro municipal. La última sección llamada Varios presenta algunas de las personas más sobresalientes del municipio ya sea que vivan en el municipio o en otras ciudades. 
De la misma forma, hay otros servicios que permiten a los usuarios obtener información acerca de cosas que pasan en su comunidad. Por ejemplo, se pudo identificar el Chat, un tablero de mensajes, fotos subidas por usuarios y un servicio de noticias. El Chat es una herramienta síncrona que permite a los miembros de la comunidad estar en contacto no sólo con otros miembros dentro del municipio sino también con aquellos que viven lejos de Arcelia. El tablero de mensajes, por otra parte, es una herramienta asíncrona que permite a los visitantes escribir mensajes cortos. Este servicio es usado principalmente para publicar mensajes cortos de saludos o preguntas a los miembros de la comunidad como este "Alguien de Arcelia que pueda darme información acerca de la Señora Natividad Flores Santos, favor de enviar cualquier respuesta a la dirección algo@yahoo.com”. Finalmente, el servicio de noticias está orientado a mantener enterados a los visitantes acerca de eventos que son parte de la vida en Arcelia como lo es el desfile de la Revolución Mexicana (20 de Noviembre), carreras de bicicletas y la conmemoración del Día de Independencia (16 de Septiembre). Por supuesto que muchos de estos eventos se levan a cabo una vez al año, pero hay otra clase de eventos que se también se publican como lo es alguna reunión de amigos en un fin de semana.

Con respecto a la audiencia del sitio Web, parece que muchas de las personas acceden de manera diaria, tomando en cuenta el número de fotos publicadas y los mensajes cortos que se publican. Aunque el sitio ha sido visitado alrededor de 642,555 desde octubre de 2002 , los números pueden ser poco engañosos ya que la compañía que lleva la cuenta de las visitas aumenta el número de visitas cada vez que se recarga la página, lo que significa que cada clic en el sitio incrementa el contador.

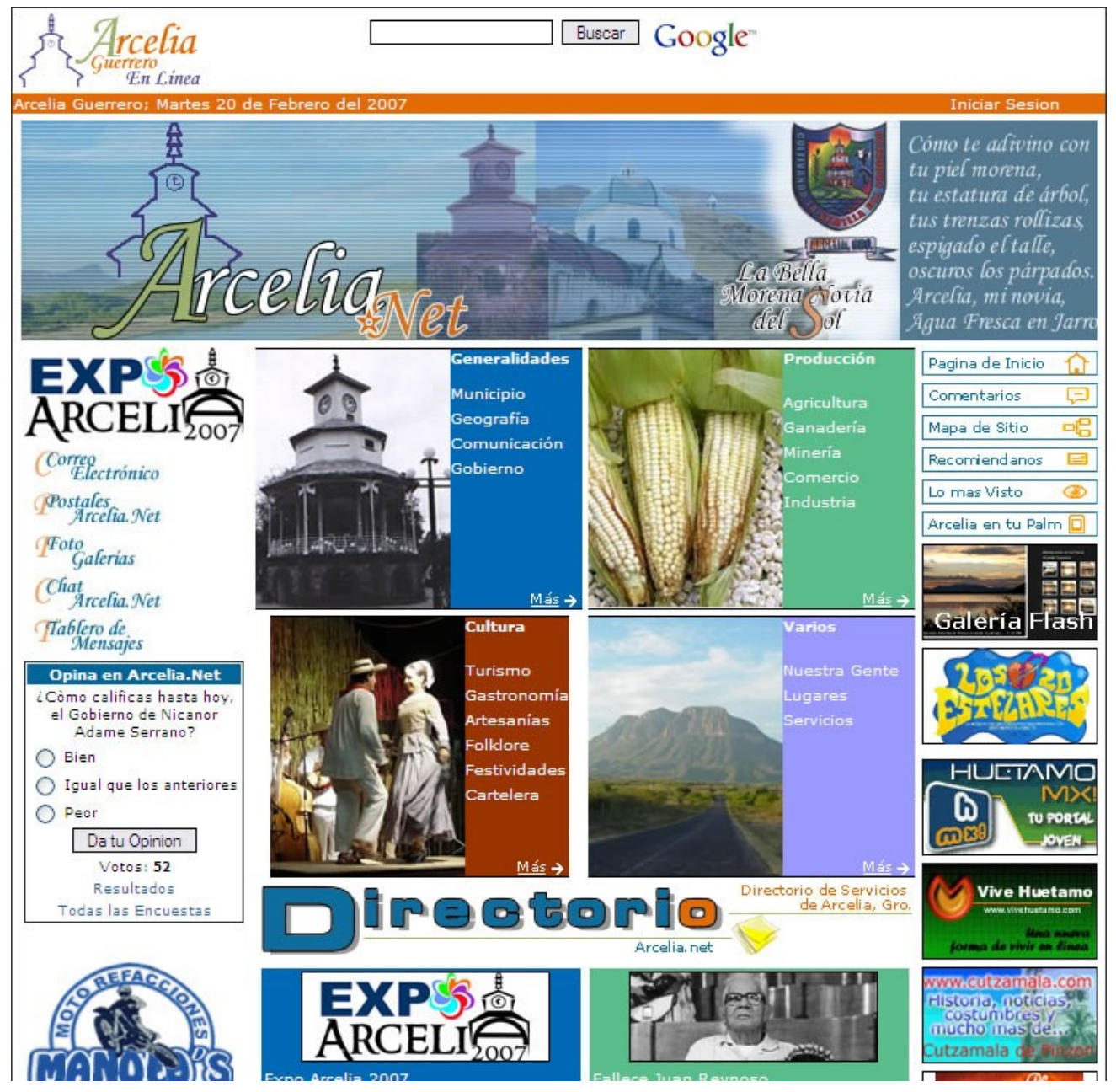

Fig 2. Captura de pantalla del sitio de Arcelia, Guerrero

\section{El sitio Web de Juchipila (http://www.juchipila.com)}

Juchipila esta localizado en el estado de Zacatecas en a parte central de México. De acuerdo con el Instituto Nacional de Estadística, Geografía e Informática (INEGI), en el 2005 la población de Juchipila era de 11,603 y el número de hogares era de 3,314. Asimismo, la penetración de computadoras era alrededor de 16.5\% (INEGI, 2005). Este municipio esta clasificado como uno con un índice de migración alto, de acuerdo a datos de CONAPO (CONAPO, 2006a).

El sitio Web de Juchipila tiene dos secciones principales: una con información local acerca de la historia de los pueblos en el municipio, festividades y leyendas regionales; la otra sección es la parte dinámica del sitio, la cual 
comprende el libro de visitas, la galería, el Chat y un directorio de usuarios. De igual forma, la página principal presenta información acerca de las condiciones climatológicas actuales en Juchipila así como una pequeña encuesta.

Una de los servicios más usados es el libro de visitas, el cual es normalmente usado para enviar mensajes a toda la comunidad, de igual forma se usa como medio de comunicación uno a uno. De hecho, también se usa como una manera de saludar a la familia y amigos tal como lo ilustra el siguiente mensaje publicado por un miembro de la comunidad viviendo en Chicago, Illinois "Me gustaría mandar saludos a toda mi familia de Juchipila, Zac... los López y Pérez por mi padre Santiago López y mi madre Julia Pérez. Podré ir a verlos el siguiente Julio en 2007. Estaremos allá para una fiesta de XV años, la Lopezada y la boda de mi hermano Jorge López... Saludos a todos y muchos besos y abrazos!”. El libro de visitas es uno de los servicios más usados así como la galería de fotos que sustenta una forma de comunicación más explicita ya que la gente comparte sus experiencias en eventos como una fiesta familiar.

Con respecto al uso del sitio, no hay estadísticas del sitio Web, al menos no estaban visibles para el publico en general. Sin embargo, el número de fotos y mensajes publicados dan una idea del nivel de actividad. La galería tienen 2,357 fotos, el libro de visitas tiene 2,008 mensajes y, finalmente, el directorio tiene 2,371 registros.

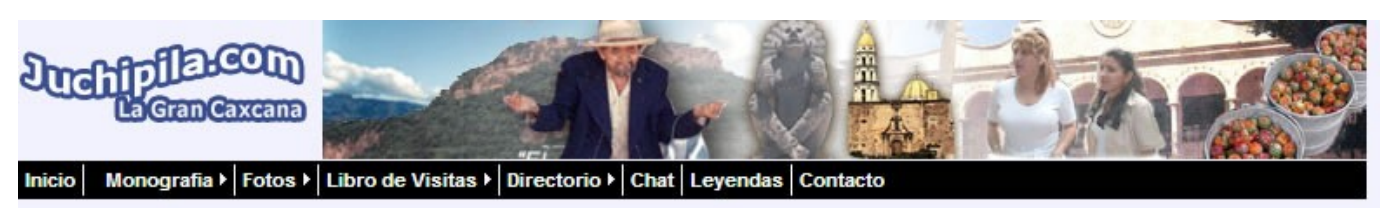

Anuncios G000000ale

Zacatecas Hotels
Reserve Online.
See Maps and
Photos Zacatecas
Hotel Discounts
BockingPage.com
Zacatecas on
Yahoo
Featured Deals
with Low Rates
Attractions,
Reviews \& Hotel
Photos
travel.yahoo.com

\section{Antique Mexico}

Quality new and

used items. Find

antique mexico

now!

inw.ebsy.com

\section{Cancun}

Beachfront $175 \mathrm{~K}$

Two Oceanfront

studios . Great buy.

Cancun Hotel

Zone, Don't miss

it!

mow. Canoun-Buyer.

Broker.com

Anunciarse en este sitio
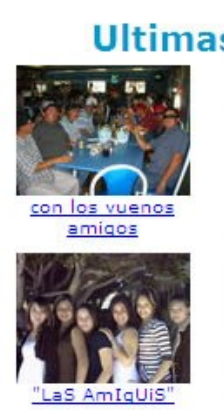

\section{(1)}

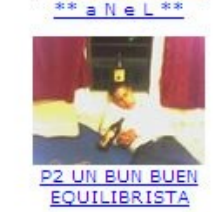

\section{Libro De Visitas}

2005 comentarios desde el Nov/08/2000 Envia tu comentario aqui

Ultimos Comentarios

Saludos a toda la gente de Juchipila! Mi nombre es Hugo Castro y soy originario de Zacatecas. Quiero pedirles una gran favor a alguien conoce a Nereida Gonzalez Castro, por favor paseme alguna informacion sobre ella o si tienen su correo mandenmelo por favoro o si le dan el mío igual se los agradeceré. Ella y yo fuimos compañeros en la facultad de derecho de la UAZ y ya van a ser casi dos años que no la veo y no se nada de ella. ASí que apelo a su buen corazón para que me ayuden y de antemano les luego.

Hugo Chugomagister@hotmail.com] 2007 6:52 PM

HOLA A TODOS Q ES LA Q AY SOLO PASABA PARA SALUDAR A MI GENTE JUCHIPILENSE Y INVITARLOS A MI SPACIO DE INTERNET

\section{Encuestas de Juchipila.com}

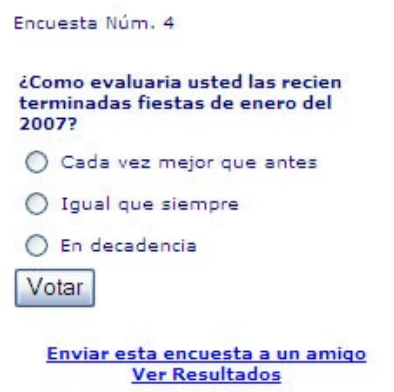

\section{El Clima hoy}

02/20/2007 03:50 AM

Estado del tiempo en Juchipila, MX a las 5:50 am CST

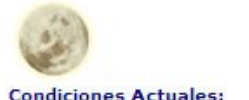

Condiciones Actuales:
Despejado, $7^{\circ} \mathrm{C}$

Pronóstico:

Mar-Soleado. Max: $30^{\circ} \mathrm{C}$ Min: $5^{\circ} \mathrm{C}$

Mie - Soleado. Max: $32^{\circ} \mathrm{C}$ Min: $6^{\circ} \mathrm{C}$

Pronóstico Completo en Yahool

Weather (Fuente: The Weather Channel)

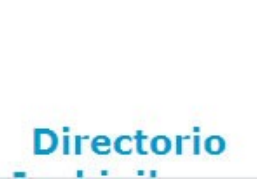

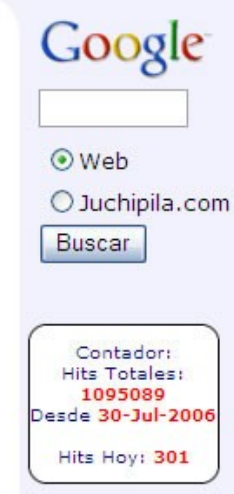

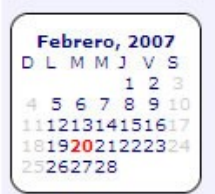

Abarrotes

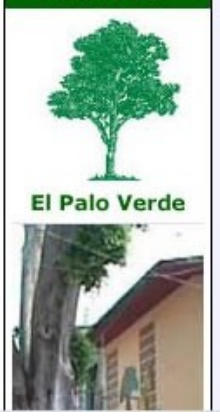

Fig. 5. Captura de pantalla del sitio Web de Juchipila, Zacatecas

\section{El sitio Web de Moyahua (http://www.moyahua.com)}

El municipio de Moyahua esta localizado también en el estado de Zacatecas. La población de Moyahua era de 4,600 en el 2005, el número de hogares ocupados era de 1,293 y el porcentaje de penetración de computadoras entre la población era de $7.35 \%$, de acuerdo a datos de INEGI (INEGI, 2005). Este municipio esta clasificado por CONAPO como uno con alto grado de migración (CONAPO, 2006a). 


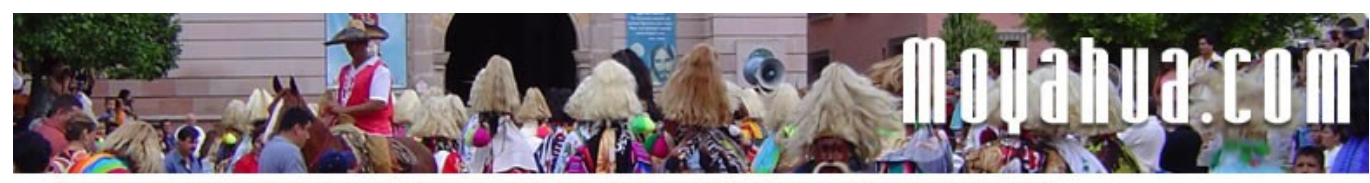

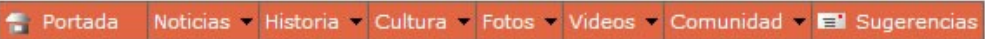

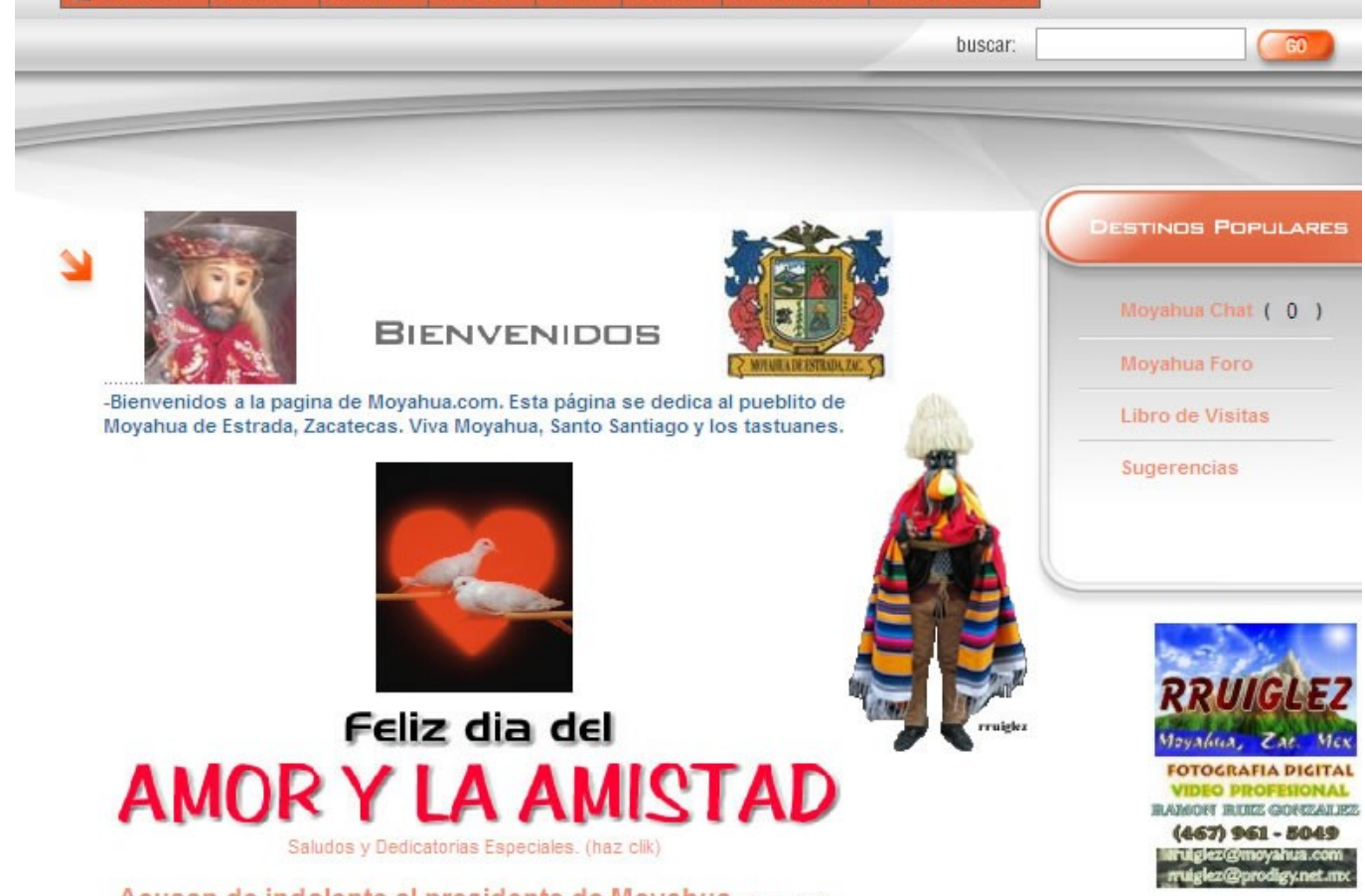

Acusan de indolente al presidente de Moyahua. (haz click)

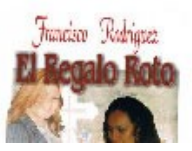

" EL REGALO ROTO"

Fig. 6. Captura de pantalla del sitio Web de Moyahua, Zacatecas (clip_image005.jpg).

El sitio Web de Moyahua cuenta con varias secciones: la sección de Noticias la cual consta de varias sub-secciones similares a las encontradas en un periódico convencional: noticias locales, eventos, entrevistas, clima y deportes. Sin embargo, todas estas secciones tratan sólo asuntos locales. Hay otras dos secciones llamadas Historia y Cultura la cual provee información histórica, información acerca de gente sobresaliente de la comunidad así como también de festividades locales. De manera similar, cuenta con una galería de video y fotografías agrupadas en álbumes fijos creados por el webmaster. Por último, hay una sección llamada Comunidad la cual comprende el Chat, foros, directorio de usuarios, comercio local, escuelas locales, bares y otras comunidades vecinas.

De acuerdo con el sitio Web, las secciones más visitadas son, por orden de aparición: el Chat, foros, libro de visitas y la de comentarios al webmaster. El foro tiene 2,233 mensajes, el libro de visitas tiene 4,252 registros; por último, hay 4,566 fotos en la galería. De acuerdo al sitio, ha habido 14,352 visitantes desde noviembre del 2003.

\section{Servicios que ofrecen y necesidades que atienden los sitios Web}

Después de analizar los sitios Web así como de clasificar los servicios que cada sitio ofrece, se llevó a cabo un análisis más detallado para caracterizar los servicios de acuerdo al fin general que persiguen y a quien están dirigidos. Se encontró que algunos de estos servicios eran para tratar de satisfacer una necesidad en particular de aquellos que viven lejos así como los que se quedan en la comunidad. Esta sección presenta la clasificación de los servicios de acuerdo a quien van dirigidos y la clase de necesidades que sustentan.

\section{Servicios ofrecidos por los sitios Web}

En estas cuatro comunidades se identificaron servicios que pueden ser clasificados en tres categorías:

\section{Servicios para unir a la comunidad}

Estos servicios se usan no sólo para contribuir al contenido del sitio Web sino también para permitir a los usuarios 
expresarse y se usan principalmente como un canal más de comunicación. Estos servicios se usan para mantener ciertos lazos con la gente de la comunidad. Incluye una gama de servicios tal como foros, galerías dinámicas, tableros de mensajes y libros de visitas.

De igual forma, esta clase de servicios permite a los que viven lejos estar al tanto de lo que pasa en la comunidad. Además, algunos usan estos servicios para enterarse de esas cosas diarias que suceden en la comunidad que ayudan a caracterizar completamente el contexto comunitario. De manera similar, los usuarios que están en la comunidad usan estos servicios para averiguar que hacen sus viejos amigos que ya no viven en la comunidad. En general, esta clase de servicios se vuelven las partes más visitadas de estos sitios.

\section{Servicios para proyectar la comunidad}

Estos servicios se usan para dar a conocer información general acerca de la comunidad. Esta clase de servicio intenta presentar las características del municipio y, enfatiza, principalmente, las particularidades de la región como lo es la cultura regional, la producción agropecuaria, cocina así como artesanías producidas en la región. Además, a veces incluyen la música típica de la región así como danzas tradicionales y fiestas tradicionales. En general, estos servicios casi siempre se leen principalmente por usuarios que visitan por primera vez el sitio Web.

\section{Servicios para obtener una imagen viva de la comunidad}

Estos servicios puede hacen experimentar al usuario la sensación de la vida cotidiana de la comunidad. La mayoría de los sitios Web presenta las condiciones meteorológicas y climáticas actuales en la comunidad, lo cual no se compara a estar físicamente allá, pero el usuario se puede dar una idea de cómo seria estar allá en ese preciso momento. Otro servicio que ofrecen algunos de los sitios es la radio local. Por último, un par de sitios han puesto cámaras en vivo en lugares públicos las cuales pueden ser controladas por los usuarios. Dicho esto, ellos pueden de hecho ver quien esta allá y que esta pasando en ese preciso momento en el lugar donde se encuentran las cámaras. Estos servicios pueden resultar muy valiosos para aquellos que viven lejos porque de alguna forma pueden 'vivir' esas pequeñas cosas que difícilmente verían en televisión o a través de una llamada de teléfono.

\section{Necesidades que sustentan los sitios Web comunitarios}

El análisis de algunos comentarios, mensajes y otras formas de contenido de algunos de los sitios Web delineo algunas de las necesidades que sustentan estos sitios Web comunitarios, las cuales se describen a continuación:

\section{Necesidad de expresar pertenencia a su comunidad}

Se encontró que algunos sitios Web sirven como un instrumento para expresar una forma de conexión y apego a la comunidad, evidenciando un sentido de pertenencia. Casi siempre, estas expresiones las hacen los usuarios cuando recién descubren el sitio Web de su comunidad ya que mandan mensajes al webmaster mostrando su alegría. De hecho, algunos de los comentarios que se analizaron mostraron que los que viven lejos encontraron al sitio como algo valioso y algo que podría fácilmente generar sentimientos de satisfacción y alegría. Los usuarios generalmente usan las herramientas que tengan a la mano para transmitir esta clase de sentimientos. Dichas herramientas se pueden encontrar en casi cualquier sitio Web comunitario en México: libro de visitas, tablero de mensajes, galería de fotos mantenida por los usuarios, mensajes rápidos y mensajes de texto SMS publicados desde el teléfono celular.

\section{Necesidad de conectarse con la vida cotidiana}

Los miembros de la comunidad necesitan un medio de comunicación donde puedan obtener las noticias más nuevas de la comunidad. En algunos de los sitios, los usuarios usan el libro de visitas o las herramientas para compartir fotos como medios directos de comunicación. También se encontró que algunas herramientas que los usuarios usan para enriquecer el contenido del sitio como lo el es servicio para compartir fotos sirven para dos cosas: 1) para conectar aquellos que viven lejos con los aspectos cotidianos de la comunidad y 2) para conectar a los que viven en la comunidad con las nuevas realidades de aquellos que han migrado. La gente de la comunidad generalmente usa este tipo de herramientas para mostrar lo que pasa en las festividades, actividades cotidianas, fiestas y reuniones, mientras que los que viven lejos lo san para mantener a su familia y amigos al tanto de lo que pasa en sus nuevos hogares. Dichos eventos incluyen graduaciones, fiestas de cumpleaños así como actividades escolares o de trabajo. Entre las herramientas que se pueden encontrar que sustentan la necesidad de conectarse con la vida cotidiana tenemos entrevistas con gente de la comunidad, las condiciones climatológicas, fotografías que se comparten, enlaces a radio y televisión locales, video en vivo así como comentarios de los usuarios de cualquier tipo. 
En general, los sitios Web estimulan la participación de los que viven lejos en eventos sociales y proyectos comunitarios como lo son la restauración de áreas o edificios públicos. Es por eso que es importante proveer los medios para que los miembros colaboren. Esto se hace enviando ideas al webmaster por correo electrónico. Sin embargo, se cree que existen otras alternativas que podrían ser exploradas como los populares Wikis, los cuales son páginas Web que pueden ser editadas por los usuarios que las visitan.

\section{Discusión}

Mucho se ha dicho acerca de la caracterización de los sitios Web, los servicios que prestan así como también las necesidades que sustentan. Sin embargo, algo que se debe notar es el nivel de presencia que estos sitios Web tienen como un todo en el contexto nacional de municipios experimentando el fenómeno de migración. Sin duda, las comunidades con más altos niveles de migración serán mas propensas a tener implementado esta clase se sitios.

Los resultados que se obtuvieron indican que cuando hablamos de presencia en línea de las comunidades, se refiere a pequeñas comunidades, donde la gente es más fácil que conozca a los demás de manera directa o indirecta. Esto puede hacer que las comunidades sean más cohesivas y más unidas y con un sentido de identidad más fuerte, lo cual puede ser un leve indicador de por qué una gran proporción de los sitios (71\%) cuentan con nombres de dominio propio.

Aunque solamente un $20 \%$ de las comunidades con alto y muy alto índice de migración tienen presencia en línea, se puede decir que estos números indican que aun así, hay un número importante de comunidades (94) que están usando el Internet de manera activa para conectar aquellos que viven lejos. Se requeriría un estudio longitudinal para medir los cambios a través del tiempo así como los efectos que tienen las nuevas tecnologías en la gente de modo que se puedan crear sitios Web como estos más fácilmente y a menor costo.

El análisis de la funcionalidad que ofrecen los sitios Web comunitarios deja en claro que los servicios reflejan, hasta cierto punto, una perspectiva más cercana a la vida en la comunidad. Se encontró que a través de los sitios Web la gente puede 1) comunicarse con la gente viviendo en la comunidad o viviendo fuera de ella, 2) compartir fotos o videos, 3) consultar información de referencia o histórica de la comunidad y 4) obtener información local actualizada. Se encontró, además, que los usuarios de estos sitios buscan la manera de mantener sus redes sociales previas así como mantenerse al tanto de lo que pasa en la comunidad, confirmando lo que otros estudios habían encontrado (Bernal, 2005; Miller and Slater, 2000). De la misma manera, se puede ver que estos sitios pueden despertar o reforzar el sentido de pertenencia y apego a la comunidad tal como en los casos de Trinidad y Eritrea presentados anteriormente (Bernal, 2005; Miller and Slater, 2000). Sin embargo, si se contrasta con esos estudios, se puede ver que el fenómeno aquí ocurre a nivel municipal y toma un sabor más local, ya que es más fácil que los usuarios se conozcan entre ellos y tengan más cosas en común. Esta cercanía entre los usuarios podría explicar en cierta forma la intensa actividad que la mayoría de estos sitios experimentan a pesar de su tamaño tan pequeño.

De igual forma, otro resultado interesante fue que estos sitios comunitarios ofrecen tres principales tipos de servicios y tienden a satisfacer, además, tres necesidades. Los principales servicios son: 1) servicios para unir a la comunidad, 2) para proyectar a la comunidad y, finalmente, 3) servicios para una imagen viva de la vida en la comunidad. En el caso de los trinitarios en diáspora, Miller y Slater (2000) encontraron que el Internet ha ayudado a reforzar las los lazos familiares. Sin embargo, en el caso de los sitios de comunidades pequeñas en México, el refuerzo se nota a nivel comunitario más que el familiar. Con respecto a las necesidades que los sitios sustentan, se encontraron tres principales: a) la necesidad de expresar apego a la comunidad, b) la necesidad de estar conectado con la vida cotidiana y c) la necesidad de contribuir y participar. Esto es muy similar a lo encontrado por Bernal (2005), quien en sus conclusiones comentó que las herramientas en línea que usaban los eritreos en diáspora sirvió para involucrarlos en asuntos nacionales por medio de discusiones en línea así como también hacerlos sentir parte de una comunidad de la que ya no eran parte físicamente.

Por último, es claro que la mayoría de estos sitios Web fueron hechos para ser usados en computadoras de escritorio. Sería muy interesante observar las oportunidades de interacción que se generarían a través de tecnologías móviles así como pantallas públicas de modo que puedan ser testigos de cosas que pasan a medida que suceden. Hay, de hecho, algunos proyectos como lo son las Pantallas Urbanas presentado por Struppek (Struppek, 2006) o las recientemente develadas por Innovation Labs, las pantallas de hechas de concreto (Innovation-Labs, 2006) que pueden servir como puntos de partida para entender los efectos que tales tecnologías pueden tener al conectar a los emigrantes con sus comunidades de origen.

\section{Conclusiones}

El incremento de la migración ha hecho que muchas comunidades se dispersen y sus miembros queden esparcidos en varias ciudades o hasta separados por fronteras. La necesidad de estar comunicado se acentúa cuando la gente se aleja de la personas que estiman. En este trabajo se presentó un análisis de los sitios Web de comunidades mexicanas de emigrantes viviendo en los EUA, con el objetivo de cuantificar la presencia de las comunidades con niveles más altos de migración. De igual forma, se intentó caracterizar el sustento que estas comunidades ofrecen a los que viven lejos de 
sus comunidades. Algunos de los servicios identificados sustentan algunas necesidades en particular como el expresar su apego a la comunidad, conectarse con la vida cotidiana así como tomar un rol activo en la vida de la comunidad. Además, algunos servicios sirven ciertos propósitos, como lo es el de reunir gente de la comunidad, proyectar la comunidad al mundo exterior y, por último, permitir a los que viven lejos ser parte de la vida cotidiana y ser testigos 'presenciales' de ciertos sucesos.

Aun se requiere un gran esfuerzo para lograr caracterizar el universo de sitios Web comunitarios en México, lo cual, de hecho es un panorama cambiante ya que nuevos sitios emergen cada día. Ademas, es importante aclarar que aun cuando este trabajo presenta resultados sólo de los municipios con alto y muy alto índice de migración, estos resultados podrían ser diferentes si se toman en cuenta los municipios con menores niveles de migración o también, buscando sitios Web no solo a nivel municipal sino a nivel comunitario (ejidos, rancherías, etc).

Tenemos la confianza de que con la incorporación gradual de tecnologías nuevas y novedosas se puede ayudar a los emigrantes a vivir mejor esas realidades duales que viven cada día así como incrementar el sentido de conexión con su comunidad de origen a través de aumentar su repertorio de comunicación. Sin embargo, se necesita un trabajo mas extenso es requerido para estudiar los efectos a largo plazo que pueden tener esa nuevas tecnologías y prácicas de acercamiento. Los esfuerzos venideros de esta investigación estarán orientados a estudiar estos efectos al mismo tiempo que los usuarios interactúan mediante novedosas tecnologías mediando sus contactos con la familia y amigos.

\section{Referencias}

AMIPCI (2007). Usuarios de Internet en México y Uso de Nuevas Tecnologías. http://www.amipci.org.mx/estudios.php.

BANXICO (2006). Family Remittances. http://www.banxico.gob.mx/polmoneinflacion/estadisticas/balanzaPagos/balanzaPagos.html.

Bernal, V. (2005). Eritrea on-line: Diaspora, cyberspace, and the public sphere. American Ethnologist 32(4): 660-675.

Castles, S. and M. J. Miller (2003). The Age of Migration: International Population Movements in the Modern World, Palgrave Macmillan.

CONAPO (2005). Population Resident in the US. http://www.conapo.gob.mx/mig_int/series/03.htm.

CONAPO (2006a). Mexico-US Migratory Intensity by Municipality. http://www.conapo.gob.mx/publicaciones/intensidadmig/CUA_AB_IIM2000.XLS.

CONAPO (2006b). Population Projections for Mexico 2000-2050. http://www.conapo.gob.mx/00cifras/5.htm.

Conway, D. and J. H. Cohen (1998). Consequences of Migration and Remittances for Mexican Transnational Communities. Economic Geography 74(1): 26-44.

Fisher, S. (1989). Homesickness, Cognition, and Health, Lawrence Erlbaum Associates.

Fox, S. and G. Livingston (2007). Latinos Online. Pew Hispanic Center and Pew Internet Project.

Gonzalez-Mendez, J. (2002). emigrantes en línea: de la nostalgia a la acción política. Masiosare Magazine, La Jornada (UNAM). Sept 15, 2002.

Hiller, H. H. and T. M. Franz (2004). New ties, old ties and lost ties: the use of the internet in diaspora. New Media \& Society 6(6): 731-752.

INEGI (2005). II Population and Households Count. http://www.inegi.gob.mx/est/contenidos/espanol/sistemas/conteo2005/localidad/iter/default.asp.

Innovation-Labs (2006). The World's first screen to be made of concrete. http://www.innovationlab.dk/sw22811.asp.

Merz, B. J. (2005). New Patterns for Mexico: Observations on Remittances, Philanthropic Giving, and Equitable Development, Harvard University Press. Miller, D. and D.

Slater (2000). The Internet: an ethnographic approach, Berg.

Salazar, G. P. (2004). Análisis crítico del Sistema Nacional e-México: la estrategia web del gobierno federal para la reducción de la brecha digital. Facultad de Ciencias Politicas y Sociales. Mexico, DF, UNAM. Master degre: 275.

Struppek, M. (2006). The social potential of Urban Screens. Visual Communication 5(2): 173.

van Tilburg, M. A. L. and A. Vingerhoets (1997). Psychological Aspects of Geographical Moves: Homesickness and Acculturation Stress, Tilburg University Press.

Walsh, E. O. (2000). The Truth About The Digital Divide. The Forrester Brief 30: 45-60. 
Zúñiga, V. and R. Hernández-León (2005). New Destinations: Mexican Immigration in the United States, Russell Sage Foundation. 